\title{
Letter REPLY Re: "Endovascular Treatment of a Symptomatic Thoracoabdominal Aortic Aneurysm by Chimney and Periscope Techniques for Total Visceral and Renal Artery Revascularization"
}

\author{
Maurizio Cariati · Umberto G. Rossi
}

Received: 17 January 2014/ Accepted: 7 February 2014/Published online: 11 April 2014

(C) Springer Science+Business Media New York and the Cardiovascular and Interventional Radiological Society of Europe (CIRSE) 2014

We appreciated the comments about the saccular pseudoaneurysm originating from the fusiform abdominal aneurysm in the Letter to the Editor from Canyigit et al. [1]. We agree with the comment of Canyigit et al. [1] that this kind of pathology is extremely rare and that penetrating atherosclerotic ulcer is the most likely underlying mechanism of its formation [1]. Contrast-enhanced multidetector computed tomography (MDCT) has become the technique of choice to evaluate aortic disease for its specificity, sensibility, and availability [2].

To our knowledge, the total number of described saccular pseudoaneurysms originating from the fusiform abdominal aneurysm has increased to six cases in total $[1,3,4]$. In our previous paper, we have not emphasized this pathological aspect, because the focus of the case report was on revascularization [3]. However, on MDCT followup after endovascular treatment the pseudoaneurysmatic sac showed a considerable reduction in volume [3].
Conflict of interest None.

\section{References}

1. Canyigit M, Kucuker A, Annac G, Hidiroglu M (2014) Saccular pseudoaneurysm originating from fusiform abdominal aortic aneurysm. Cardiovasc Intervent Radiol. doi:10.1007/s00270-0140877-2

2. Chiu KWH, Lakshminarayan R, Ettles DF (2013) Acute aortic syndrome: CT findings. Clin Radiol 68:741-748

3. Cariati M, Mingazzini P, Dallatana R, Rossi UG, Settembrini A, Santuari D (2014) Endovascular treatment of a symptomatic thoracoabdominal aortic aneurysm by chimney and periscope techniques for total visceral and renal artery revascularization. Cardiovasc Intervent Radiol 37(1):251-256

4. Lindblad B, Holst J, Kölbel T, Ivancev K (2008) What to do when evidence is lacking-implications on treatment of aortic ulcers, pseudoaneurysms and aorto-enteric fistulae. Scand J Surg 97: $165-173$
M. Cariati $(\bowtie) \cdot$ U. G. Rossi

Division of Radiology and Interventional Radiology,

Department of Diagnostic Sciences, San Carlo Borromeo

Hospital, Via Pio II, 3, 20153 Milan, Italy

e-mail: cariati.maurizio@sancarlo.mi.it 